\title{
DIMENSIONAMENTO DE MOTORES PARA O BOMBEAMENTO DE ÁGUA
}

\section{DELLY OLIVEIRA FILHO ${ }^{1}$, MARCOS C. RIBEIRO ${ }^{2}$, EVERARDO C. MANTOVANI ${ }^{3}$, ANTÔNIO A. SOARES ${ }^{4}$, HAROLDO C. FERNANDES ${ }^{5}$}

RESUMO: O dimensionamento de motores com potência acima da necessária acarreta maior custo inicial, menor rendimento e menor fator de potência. Desde 1996, que se fabricam no Brasil motores elétricos com fator de serviço maior do que a unidade, obedecendo a valores prescritos em norma. O fator de serviço informa a potência disponível do motor em regime contínuo para as condições de carga nominal. A literatura técnica de dimensionamento de motores para acionamento de bombas hidráulicas indica a necessidade da adoção de acréscimos na potência dos motores. Tanto o fator de serviço, quanto o acréscimo na potência constituem fatores de segurança para o dimensionamento. Estes fatores de segurança têm a função de suprir maior demanda de potência devido a variações nas condições de trabalho, como curvas características da bomba ou do motor, e a qualidade de energia, como oscilação e desequilíbrio do sinal de tensão. Conclui-se que não seja utilizado acréscimo na potência no dimensionamento de motores com fator de serviço maior do que a unidade para acionamento de bomba hidráulica, tendo em vista que o superdimensionamento pode acarretar redução de rendimento do sistema e maior custo inicial. O dimensionamento que considera, simultaneamente, os dois fatores de segurança, implica superdimensionamento de $15 \%$ a $88 \%$.

PALAVRAS-CHAVE: fator de segurança, motores elétricos, bombeamento.

\section{SIZING MOTORS FOR WATER PUMPING}

ABSTRACT: Sizing motors with power above the required implies in higher initial cost, lower performance and lower power factor. Since 1996, that electrical motor is built in Brazil with service factors greater than the unity, obeying the standard values. The service factor informs the motor's available power in continuous regime under nominal condition. The technical literature for sizing motors to operate hydraulic pumps indicates the need of adoption of sizing factors in the motor's power. Both the service factor and the sizing factor are security factors to size motors. These safety factors have the function to supply greater power demand due to changes in the working conditions such as, pump or motor characteristic curves, or energy quality like voltage imbalance or oscillation. The conclusion is that there is no need to use sizing factor for rating motors to drive hydraulic pump, because oversize may lead in performance decrease and greater initial costs. The size that considers both the security factors implies in an over sizing from $15 \%$ to $88 \%$.

KEYWORDS: security factors, electrical motors, pumping.

\section{INTRODUÇÃO}

O motor elétrico é o equipamento responsável pela transformação da energia elétrica em energia mecânica, sendo, na maioria dos segmentos econômicos rurais e industriais, a principal forma de uso final da energia elétrica. No Brasil, os motores representam mais de $50 \%$ do uso final da energia elétrica da indústria. Desde o início de sua utilização, houve redução de seu peso e aumento do seu rendimento, especialmente pela melhoria da tecnologia dos materiais isolantes, dos materiais magnéticos e do projeto como um todo.

\footnotetext{
${ }^{1}$ Eng $^{\mathrm{o}}$ Eletricista, Ph.D. Professor do Departamento de Engenharia Agrícola da UFV, delly@ufv.br.

${ }^{2} \mathrm{Eng}^{\mathrm{O}}$ Agrícola, D.S., Instituto Federal do Sul de Minas - Câmpus Inconfidentes, marcos.ribeiro@ifs.ifsuldeminas.edu.br.

${ }^{3}$ Eng $^{0}$ Agrícola, D.S., Departamento de Engenharia Agrícola da UFV, everardo@ufv.br.

${ }^{4}$ Eng $^{\mathrm{O}}$ Agrícola, Ph.D., Departamento de Engenharia Agrícola da UFV, aasores@ufv.br.

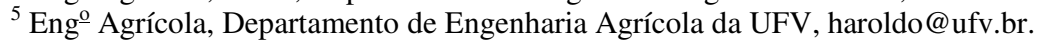

Recebido pelo Conselho Editorial em: 17-8-2009

Aprovado pelo Conselho Editorial em: 26-10-2010
} 
Para o dimensionamento da potência requerida de um motor, os fatores de segurança ou de acréscimo são, em geral, considerados como garantia de sucesso de seu funcionamento. O dimensionamento de sistema de bombeamento que depende de diversos pontos de determinação de potência, faz uso desse fator de segurança. Para o dimensionamento da potência necessária de um motor, as folgas técnicas vão-se acumulando, podendo gerar um superdimensionamento, com maior emprego de capital e desperdício de energia, devido ao baixo rendimento e ao baixo fator de potência.

O rendimento e o fator de potência variam com a carga do motor. As curvas características dos motores indicam os valores típicos de rendimento e de fator de potência em função da carga. Normalmente, a curva característica de rendimento de um motor de indução apresenta valores maiores quando a carga do motor se situa entre $75 \%$ e $100 \%$ de sua capacidade nominal, enquanto seu fator de potência atinge o valor máximo com $100 \%$ a $125 \%$ de carregamento nominal. Portanto, com um carregamento abaixo de $75 \%$ do nominal, os motores elétricos requerem, proporcionalmente, maior quantidade de energia elétrica para produzir a mesma energia mecânica. As perdas são mais baixas em motores de alto rendimento (WEG, 2000).

Os motores elétricos têm capacidade de suportar cargas de trabalho superiores à nominal. A percentagem de sobrecarga que um motor elétrico suporta é chamada de fator de serviço (FS).

O fator de serviço é um multiplicador que, quando aplicado à potência nominal do motor, indica a carga que pode ser acionada continuamente sob tensão e frequência nominais e com limite de elevação da temperatura de enrolamento. Os valores de rendimento, de fator de potência e de rotação podem diferir dos nominais, porém o conjugado e a corrente com rotor bloqueado e o conjugado máximo permanecem inalterados. A utilização do fator de serviço implica vida útil inferior àquela do motor com carga nominal (ABNT, 1996). Assim, o fator de serviço representa uma reserva de potência que o motor possui, e que pode ser usada em regime contínuo.

O fator de serviço não deve ser confundido com a sobrecarga momentânea do motor, a qual vale por curtos períodos de tempo. De acordo com a norma brasileira, motores de aplicação geral devem suportar sobrecarga de torque de até $60 \%$ acima do valor nominal durante 15 segundos.

O motor de alto rendimento apresenta vantagens sobre o motor convencional, como: (i) redução do consumo de energia elétrica; (ii) fator de potência mais elevado, e (iii) menor temperatura de operação (ELETROBRÁS, 1998). O desenvolvimento do presente trabalho objetivou realizar uma avaliação dos fatores de segurança aplicados ao dimensionamento de motobombas para irrigação.

Os objetivos específicos foram: (i) determinar o índice de carregamento, a potência nominal e o rendimento de sistemas de bombeamento de água para irrigação com e sem os fatores de segurança propostos pela literatura; e (ii) determinar a diferença entre os gastos com energia elétrica (demanda de potência e consumo) para sistemas de bombeamento para irrigação com e sem os fatores de segurança propostos pela literatura.

\section{MATERIAL E MÉTODOS}

Este trabalho foi desenvolvido no Laboratório de Energia do Departamento de Engenharia Agrícola da Universidade Federal de Viçosa, em Viçosa-MG. Foram utilizadas normas regulamentadoras para motores (ABNT, 1996), catálogos eletrônicos de motores e o programa para seleção de bombas agrícolas (SANTOS, 2001). O programa AGRIBOMBAS (2000) é um gerenciador eletrônico para escolha da bomba mais eficiente e contém dados de cinco fabricantes de bombas (EH, Mark Peerless, KSB, Imbil e Schneider), com três rotações diferentes, podendo selecionar até dez bombas distintas que melhor otimizem a escolha e as curvas características dos motores elétricos (WEG, 2000). 


\section{Análise técnica}

Os fatores técnicos de dimensionamento da potência de bombas e motores para sistemas de bombeamento de água são dependentes da altura manométrica, da vazão e do rendimento da bomba e do motor.

As bombas usadas para irrigação são, em sua maioria, do tipo centrífuga de eixo horizontal. Nos projetos de irrigação, as bombas são instaladas, em geral, acima do nível do poço de sucção.

As metodologias para a escolha das potências nominais foram as comercialmente disponíveis para estudo dos pontos críticos (mínima potência mecânica disponível).

Em geral, os projetistas dimensionam o rotor da bomba acima do ideal para a vazão de projeto e um acréscimo na potência do motor para se poder ajustar a algumas indeterminações no sistema.

A vazão necessária de água pode ser expressa pela Equação 1.

$$
\mathrm{Q}=10 \frac{\mathrm{A} \mathrm{L}}{\mathrm{h}}
$$

em que,

$\mathrm{Q}$ - vazão necessária, $\mathrm{m}^{3} \mathrm{~h}^{-1}$;

A - área a ser irrigada, ha;

$\mathrm{L}_{\mathrm{i}}$ - lâmina de água para irrigação a ser aplicada, $\mathrm{mm} \mathrm{dia}^{-1}$, e

$\mathrm{H}$ - número de horas de funcionamento diário.

A potência absorvida na bomba pode ser expressa pelas eqs.(2a) e (2b):

$$
\begin{aligned}
& \mathrm{Pa}_{\mathrm{kW}}=\frac{\mathrm{Q} \mathrm{H_{ \text {man } }}}{367 \eta_{\mathrm{b}}} \\
& \mathrm{Pa}_{\mathrm{cv}}=\frac{\mathrm{Q} \mathrm{H_{ \text {man } }}}{270 \eta_{\mathrm{b}}}
\end{aligned}
$$

em que,

$\mathrm{Pa}_{\mathrm{kw}}$ - potência absorvida na bomba, $\mathrm{kW}$;

$\mathrm{Pa}_{\mathrm{cv}}$ - potência absorvida na bomba, cv;

$\mathrm{Q}$ - vazão bombeada, $\mathrm{m}^{3} \mathrm{~h}^{-1}$;

$\mathrm{H}_{\text {man }}$ - altura manométrica, mca, e

$\eta_{b}$ - eficiência da bomba, em decimal.

Se a transmissão de potência for por meio de eixo direto, a potência fornecida pelo motor será praticamente igual à demandada pela bomba.

A potência elétrica necessária ao sistema é expressa pelas eq.(3a) e (3b):

$$
\begin{aligned}
& P_{\mathrm{kW}}=\frac{\mathrm{Q} \mathrm{H}_{\mathrm{man}}}{367 \eta_{\mathrm{b}} \eta_{\mathrm{m}}} \\
& \mathrm{P}_{\mathrm{cv}}=\frac{\mathrm{Q} \mathrm{H}_{\mathrm{man}}}{270 \eta_{\mathrm{b}} \eta_{\mathrm{m}}}
\end{aligned}
$$

em que,

$\mathrm{P}_{\mathrm{kw}}$ - potência elétrica necessária ao sistema, $\mathrm{kW}$;

$\mathrm{P}_{\mathrm{cv}}$ - potência elétrica necessário sistema, cv;

$\eta_{\mathrm{b}}$ - rendimento ou eficiência da bomba, em decimal, e

$\eta_{\mathrm{m}}$ - rendimento ou eficiência do motor, em decimal. 
Os rendimentos dos motores elétricos estudados foram escolhidos para funcionarem no limite do regime de trabalho que o fabricante da bomba determinou, sem interferência em seu rendimento, e submetidos à carga de trabalho, determinando-se o índice de carregamento (Ic) da potência fornecida em relação à nominal. Com o Ic, determinou-se o rendimento por meio da curva característica do motor, como pode ser visto na Figura 1.

O rendimento do motor $\left(\eta_{\mathrm{m}}\right)$ é calculado pela eq.(4):

$$
\eta_{\mathrm{m}}=\frac{\mathrm{P}_{\mathrm{m}}}{\mathrm{P}_{\mathrm{e}}} 100
$$

em que,

$\eta_{\mathrm{m}}$ - rendimento do motor;

$\mathrm{P}_{\mathrm{m}}$ - potência mecânica, $\mathrm{kW}$, e

$\mathrm{P}_{\mathrm{e}}$ - potência consumida, $\mathrm{kW}$.
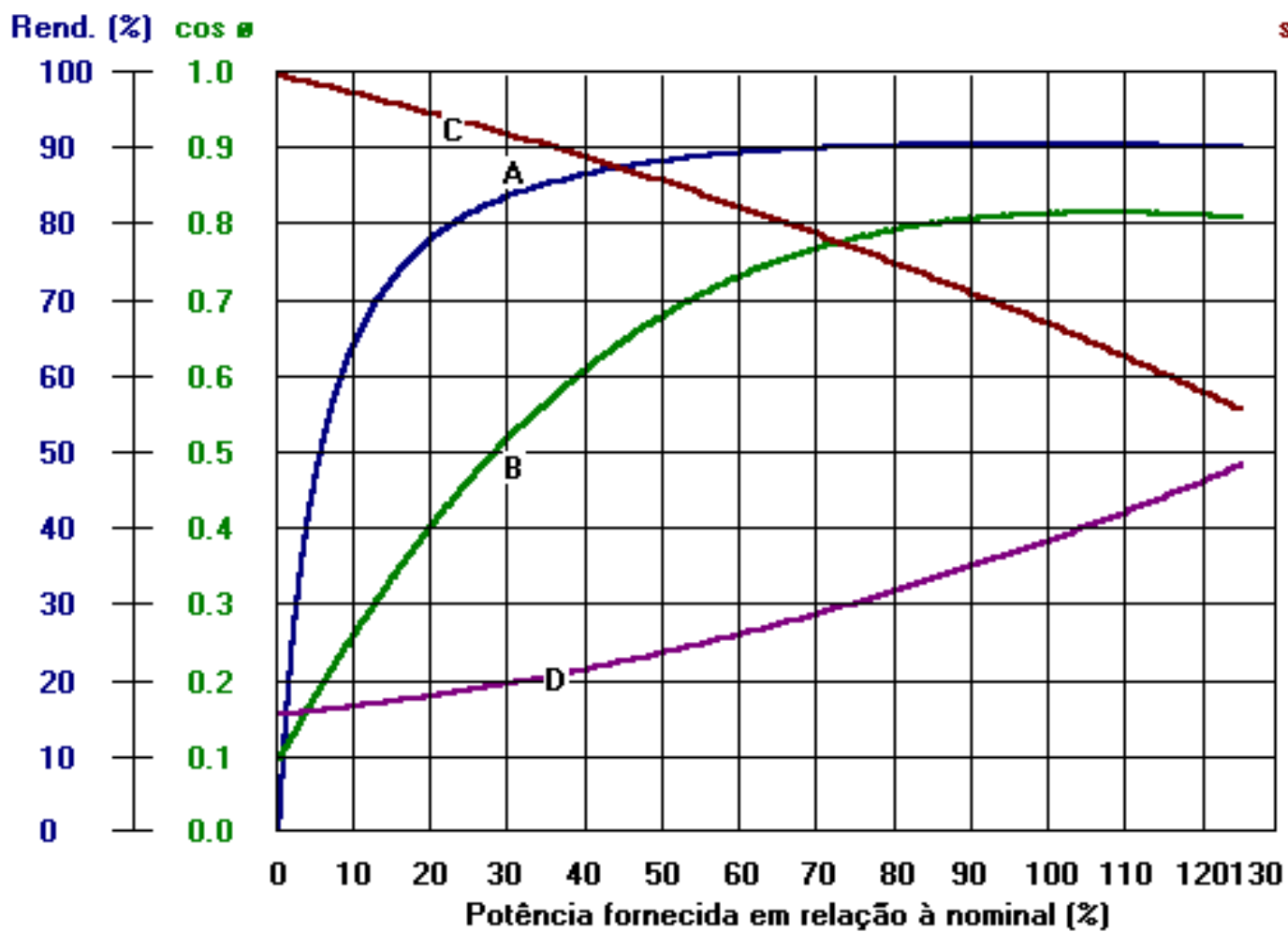

$s[\%] \quad$ Corrente [A]
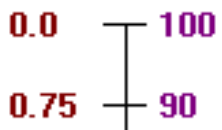

$1.5-80$

$2.25-70$

$3.0-60$

$3.75+50$

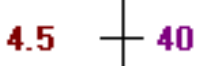

$5.75+30$

$6.0-20$

$6.75+10$

$7.5 \perp_{0}$
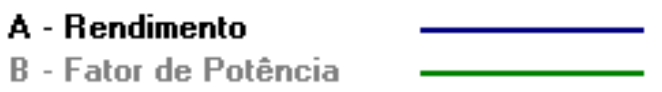

C - Escorregamento

D - Corrente

Fonte: WEG (2000).

FIGURA 1. Curvas características típicas de motor elétrico. Electric motor typical characteristics curves.

Os rendimentos das bombas centrífugas foram determinados por meio das curvas características das bombas que apresentam as relações entre altura manométrica e vazão, e potência mecânica exigida e vazão para diferentes diâmetros, rotações e modelos.

Escolheu-se a bomba centrífuga por meio do programa Agribombas, que seleciona os melhores modelos de bomba de acordo com a vazão e a altura manométrica em função do critério de máximo rendimento. Com relação ao fator de segurança nos motores, a literatura técnica sobre dimensionamento de sistema de irrigação recomenda acréscimo na potência instalada em função da potência absorvida pela bomba, conforme mostra a Tabela 1. 
Uma das razões que levaram ao uso do fator de segurança foi a não uniformidade do sistema elétrico, cuja variação de tensão pode comprometer o desempenho dos motores.

O fator de serviço (FS) aplicado e os valores recomendados de acréscimos na potência de motores elétricos para o acionamento de bombas hidráulicas recomendada pela literatura, em função da potência nominal do motor, são mostrados na Tabela 1.

TABELA 1. Valores do fator de serviço e recomendação de acréscimo na potência de motores elétricos para o acionamento de bombas hidráulicas. Service factor values and recommended electric motors power increase to activate the hydraulic pumps.

\begin{tabular}{|c|c|c|c|c|c|c|}
\hline \multicolumn{2}{|c|}{$\begin{array}{c}\text { Potência Nominal } \\
\text { do Motor }\end{array}$} & \multicolumn{4}{|c|}{ Fator de Serviço - FS* } & \multirow{3}{*}{$\begin{array}{c}\text { Acréscimo na Potência } \\
\text { Demandada } * * \\
\%\end{array}$} \\
\hline \multirow{2}{*}{$\mathrm{kW}$} & \multirow{2}{*}{$\mathrm{cv}$} & \multicolumn{4}{|c|}{ Número de Pólos } & \\
\hline & & 2 & 4 & 6 & 8 & \\
\hline 0,037 & $1 / 20$ & 1,40 & 1,40 & 1,40 & 1,40 & 30 \\
\hline 0,060 & $1 / 12$ & 1,40 & 1,40 & 1,40 & 1,40 & 30 \\
\hline 0,090 & $1 / 8$ & 1,40 & 1,40 & 1,40 & 1,40 & 30 \\
\hline 0,120 & $1 / 6$ & 1,35 & 1,35 & 1,35 & 1,35 & 30 \\
\hline 0,180 & $1 / 4$ & 1,35 & 1,35 & 1,35 & 1,35 & 30 \\
\hline 0,250 & $1 / 3$ & 1,35 & 1,35 & 1,35 & 1,35 & 30 \\
\hline 0,370 & $1 / 2$ & 1,25 & 1,25 & 1,25 & 1,15 & 30 \\
\hline 0,550 & $3 / 4$ & 1,25 & 1,25 & 1,15 & 1,15 & 30 \\
\hline 0,750 & 1 & 1,25 & 1,15 & 1,15 & 1,15 & 30 \\
\hline 1,100 & 1,5 & 1,15 & 1,15 & 1,15 & 1,15 & 30 \\
\hline 1,470 & 2 & 1,15 & 1,15 & 1,15 & 1,15 & 30 \\
\hline 2,210 & 3 & 1,15 & 1,15 & 1,15 & 1,15 & 25 \\
\hline 2,940 & 4 & 1,15 & 1,15 & 1,15 & 1,15 & 25 \\
\hline 3,680 & 5 & 1,15 & 1,15 & 1,15 & 1,15 & 25 \\
\hline 4,420 & 6 & 1,15 & 1,15 & 1,15 & 1,15 & 20 \\
\hline 5,520 & 7,5 & 1,15 & 1,15 & 1,15 & 1,15 & 20 \\
\hline 7,360 & 10 & 1,15 & 1,15 & 1,15 & 1,15 & 20 \\
\hline 9,200 & 12,5 & 1,15 & 1,15 & 1,15 & 1,15 & 15 \\
\hline 11,040 & 15 & 1,15 & 1,15 & 1,15 & 1,15 & 15 \\
\hline 14,720 & 20 & 1,15 & 1,15 & 1,15 & 1,15 & 15 \\
\hline 18,400 & $\begin{array}{l}25 \mathrm{em} \\
\text { diante }\end{array}$ & 1,15 & 1,15 & 1,15 & 1,15 & 10 \\
\hline
\end{tabular}

Nota: o valor do FS de 1,15 aplica-se somente às categorias $\mathrm{N}$ e $\mathrm{H}$, no caso de motores com rotor em gaiola polifásicos (ABNT, 1996). * ABNT 7094 (1996), ** Fator de segurança (BERNARDO et al., 2005).

Na Tabela 1, observa-se que, para cada potência nominal dos motores, os fatores de serviço (FS) têm uma variação a mais quando se utiliza número de polos, para as potências entre 0,37 e $0,75 \mathrm{~kW}$. Os valores dos acréscimos na potência demandada recomendados pela literatura servem para qualquer número de polos usados nos motores, ou seja, não há divisão quanto à polaridade.

A norma da Associação Brasileira de Normas Técnicas (ABNT), NBR 7094, define o fator de serviço (FS) como fator multiplicador da potência nominal do motor elétrico, indica a carga que pode ser acionada continuamente sob tensão e frequência nominais, com limite de elevação da temperatura do enrolamento.

\section{Análise econômica}

A estrutura das informações foram baseadas em duas faixas de horas de funcionamento (1.000 e $4.000 \mathrm{~h}$ ), uma lâmina de água para irrigação $\left(5 \mathrm{~mm} \mathrm{dia}^{-1}\right)$, duas pressões manométricas (196 e $392 \mathrm{kPa})(20$ e $40 \mathrm{mca})$ e 12 motores com potências diferenciadas $(0,5 ; 1,0 ; 1,5 ; 2,0 ; 3,0 ; 5,0 ; 7,5$; 10,$0 ; 15,0 ; 20,0 ; 50,0 ;$ e 125,0 cv). Foi utilizada a tarifa verde na análise econômica para, assim, auxiliar nas decisões de como melhor utilizar os recursos disponíveis, sem perda de qualidade dos 
resultados. Os motores empregados na simulação foram da linha alto rendimento, os quais possuem o certificado do ELETROBRÁS (1998).

O dimensionamento de motores requer o conhecimento se o uso é contínuo ou não e o número de horas de funcionamento. Deve ser considerado, também, que a demanda de potência e o rendimento diminuem à medida que o número de horas de funcionamento aumenta. Portanto, existe um limite para o aumento do número de horas de funcionamento anual para a otimização econômica.

\section{RESULTADOS E DISCUSSÃO}

\section{Análise técnica}

No Tabela 2, apresentam-se a potência de motores para acionamento de bomba e a folga percentual, considerando somente o fator de serviço (método 1) e o dimensionamento mais o fator de serviço indicados pela literatura (método 2).

A última coluna da Tabela 2 mostra a folga dos motores quando usados na potência demandada pela bomba. Observa-se, nessa Tabela, que a folga disponível da potência nominal pode chegar a valores de até $88 \%$, que são considerados extremamente elevados para motores com potências menores, quando se utiliza o acréscimo sugerido pela literatura técnica de irrigação, além do fator de serviço.

As potências demandadas pelas bombas apresentadas na Tabela 2 foram calculadas conforme o projeto hidráulico simulado no uso de potências comerciais para estudo dos pontos críticos, utilizando-se de pressões de 196 e $392 \mathrm{kPa}$ (20 e $40 \mathrm{mca}$ ), número de horas de funcionamento de 1.000 e de 4.000 horas anuais e lâmina de água para irrigação de $5 \mathrm{~mm} \mathrm{dia}^{-1}$.

TABELA 2. Potência de motores elétricos para acionamento de bombas e folga, considerando somente o fator de serviço (método 1) e o fator de serviço mais os fatores de acréscimo de dimensionamento (método 2). Electric motor power to activate pumps and clearance considering only the service factor (method 1) and the service factor plus the increasing factors to sizing (method 2 ).

\begin{tabular}{|c|c|c|c|c|}
\hline \multirow{2}{*}{$\begin{array}{c}\text { Potência Demandada } \\
\text { pela Bomba } \\
\mathrm{kW}(\mathrm{cv})\end{array}$} & \multicolumn{2}{|c|}{$\begin{array}{l}\text { Potência Demandada do Motor } \\
\text { para Acionamento da Bomba } \\
\text { kW (cv) }\end{array}$} & \multicolumn{2}{|c|}{$\begin{array}{c}\text { Folga Disponível da Potência } \\
\text { Nominal com FS } \\
\%\end{array}$} \\
\hline & Método 1 & Método 2 & Método 1 & Método 2 \\
\hline $0,37(0,50)$ & $0,46(0,63)$ & $0,69(0,94)$ & 25 & 88 \\
\hline $0,75 *(1,00)$ & $0,92(1,25)$ & $1,38(1,88)$ & 25 & 88 \\
\hline $0,75 * *(1,00)$ & $0,85(1,15)$ & $1,27(1,73)$ & 15 & 73 \\
\hline $1,10(1,50)$ & $1,27(1,73)$ & $1,69(2,30)$ & 15 & 53 \\
\hline $1,50(2,00)$ & $1,69(2,30)$ & $2,54(3,45)$ & 15 & 73 \\
\hline $2,20(3,00)$ & $2,54(3,45)$ & $3,39(4,60)$ & 15 & 53 \\
\hline $3,70(5,00)$ & $4,23(5,75)$ & $6,35(8,63)$ & 15 & 73 \\
\hline $5,50(7,50)$ & $6,35(8,63)$ & $8,46(11,50)$ & 15 & 53 \\
\hline $7,50(10,00)$ & $8,46(11,50)$ & $10,58(14,38)$ & 15 & 44 \\
\hline $11,00(15,00)$ & $12,70(17,25)$ & $16,93(23,00)$ & 15 & 53 \\
\hline $15,00(20,00)$ & $16,93(23,00)$ & $21,16(28,75)$ & 15 & 44 \\
\hline $37,00(50,00)$ & $42,32(57,50)$ & $50,78(69,00)$ & 15 & 38 \\
\hline $90,00(125,00)$ & $105,80(143,75)$ & $126,96(172,50)$ & 15 & 38 \\
\hline $110,00(150,00)$ & $126,96(172,50)$ & $148,12(201,25)$ & 15 & 34 \\
\hline $130,00(175,00)$ & $148,12(201,25)$ & $169,28(230,00)$ & 15 & 31 \\
\hline $150,00(200,00)$ & $169,28(230,00)$ & $211,60(287,50)$ & 15 & 44 \\
\hline
\end{tabular}

Obs.:(*) Potência demandada para dois polos, (**) Potência demandada para quatro polos. 
Os valores encontrados da folga pelo método 1 foram obtidos utilizando os dados de FS fornecidos pela Tabela 1, multiplicados pela potência demandada pela bomba, e os valores da folga encontrados pelo método 2 foram obtidos pela potência comercial imediatamente acima do calculado no dimensionamento e multiplicado pelo FS correspondente à potência utilizada. Exemplo: a potência de um motor de $3,70 \mathrm{~kW}(5 \mathrm{cv})$, multiplicada pelo acréscimo de $25 \%$ indicado pela literatura, resultará $4,63 \mathrm{~kW}(6,3 \mathrm{cv})$ de potência.

Como este motor não existe no mercado, compra-se o motor de potência imediatamente superior, $5,52 \mathrm{~kW}(7,5 \mathrm{cv})$, que multiplicado ao FS correspondente de 1,15 terá o resultado de $6,35 \mathrm{~kW}(8,63 \mathrm{cv})$, apresentando folga de $73 \%$.

Nas Tabelas 3 e 4, comparam-se a potência nominal do motor, o índice de carregamento, o rendimento do motor, a potência mecânica disponível e a potência elétrica útil necessária ao sistema, para as duas pressões (20 e $40 \mathrm{mca}$ ), usando o fator de serviço (FS) com os dados fornecidos pela literatura técnica de irrigação.

TABELA 3. Características técnicas de bombas e de motores elétricos com e sem acréscimos na potência em função da vazão para pressão de $196 \mathrm{kPa}(20 \mathrm{mca})$. Specifications of pump and electric motors with and without increases in power as a function of the flow for a pressure of $196 \mathrm{kPa}(20 \mathrm{~m})$.

\begin{tabular}{|c|c|c|c|c|c|c|c|c|c|}
\hline 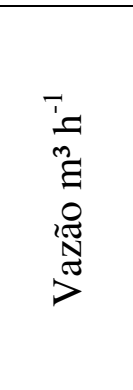 & 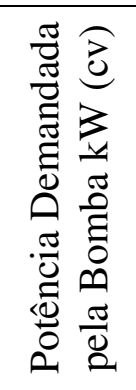 & 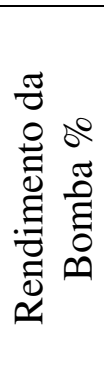 & $\begin{array}{l}\frac{n}{0} \\
0 \\
0 \\
0 \\
0 \\
\stackrel{0}{0} \\
\vdots \\
\vdots\end{array}$ & $\frac{0}{\frac{0}{0}}$ & $\begin{array}{c}\text { Potência } \\
\text { Nominal } \\
\text { do Motor } \\
(\mathrm{kW})\end{array}$ & $\begin{array}{c}\text { Índice } \\
\text { de } \\
\text { Carregamento } \\
(\%)\end{array}$ & $\begin{array}{c}\text { Rendimento } \\
\text { do } \\
\text { Motor } \\
(\%)\end{array}$ & $\begin{array}{c}\text { Potência } \\
\text { Mecânica } \\
\text { Disponível } \\
\text { Utilizando } \\
\text { FS } \\
(\mathrm{kW})\end{array}$ & $\begin{array}{c}\text { Potência } \\
\text { Elétrica Útil } \\
\text { Necessária } \\
\text { ao Sistema } \\
\text { na } \\
\text { Condição } \\
\text { Nominal } \\
(\mathrm{kW})\end{array}$ \\
\hline \multirow{2}{*}{3,1} & 0,37 & \multirow{2}{*}{46,1} & \multirow{2}{*}{4} & $1 *$ & 0,37 & 100,0 & 74,1 & 0,46 & 0,50 \\
\hline & $(0,5)$ & & & $2 *$ & 0,55 & 66,7 & 75,8 & 0,69 & 0,49 \\
\hline \multirow{2}{*}{14,3} & 1,1 & \multirow{2}{*}{70,9} & \multirow{2}{*}{2} & 1 & 1,1 & 100,0 & 86,7 & 1,27 & 1,27 \\
\hline & $(1,5)$ & & & 2 & 1,5 & 75,0 & 83,6 & 1,69 & 1,32 \\
\hline \multirow{2}{*}{26,4} & 2,2 & \multirow{2}{*}{65,1} & \multirow[b]{2}{*}{4} & 1 & 2,2 & 100,0 & 87,0 & 2,54 & 2,54 \\
\hline & $(3,0)$ & & & 2 & 3,0 & 75,0 & 86,0 & 3,39 & 2,57 \\
\hline \multirow{2}{*}{80,0} & 5,5 & \multirow{2}{*}{79,0} & \multirow{2}{*}{4} & 1 & 5,5 & 100,0 & 89,5 & 6,35 & 6,17 \\
\hline & $(7,5)$ & & & 2 & 7,5 & 75,0 & 90,2 & 8,46 & 6,12 \\
\hline \multirow{2}{*}{163,1} & 11,0 & \multirow{2}{*}{80,5} & \multirow{2}{*}{6} & 1 & 11,0 & 100,0 & 91,3 & 12,70 & 12,09 \\
\hline & $(15,0)$ & & & 2 & 15,0 & 75,0 & 91,4 & 16,93 & 12,08 \\
\hline \multirow{2}{*}{550,5} & 37,0 & \multirow{2}{*}{81,6} & \multirow[b]{2}{*}{6} & 1 & 37,0 & 100,0 & 93,6 & 42,32 & 39,32 \\
\hline & $(50,0)$ & & & 2 & 45,0 & 83,3 & 93,6 & 50,78 & 39,32 \\
\hline \multirow{2}{*}{$1.301,2$} & 90,0 & \multirow{2}{*}{77,1} & \multirow[b]{2}{*}{4} & 1 & 90,0 & 100,0 & 94,4 & 105,80 & 97,46 \\
\hline & $(125,0)$ & & & 2 & 110,0 & 83,3 & 94,9 & 126,96 & 96,94 \\
\hline
\end{tabular}

Nota: Tanto o método 1 quanto o método 2 consideraram motores elétricos com fator de serviço conforme ABNT 7094, ou seja, sempre maiores do que 1,00. (*) Método 1 - Dimensionamento de força motriz sem índices ou fatores de segurança que acrescentem a potência demandada pela bomba. (**) Método 2 - Dimensionamento de força motriz com índice ou fator de segurança que acrescentem a potência demandada pela bomba (BERNARDO et al., 2005).

A potência nominal dos motores, pelo método 1, é a potência demandada pela bomba em um mesmo eixo, e para o método 2, a potência comercialmente disponível acima da potência da bomba, utilizando o acréscimo da literatura técnica. $\mathrm{O}$ índice de carregamento é a relação entre a potência mecânica demandada pela bomba e a potência nominal do motor, em percentagem que, para o método 1 , foi em $100 \%$ e, para o método 2, atinge o máximo de $83,3 \%$. 
Esse baixo índice pode ser analisado como motor com folga, podendo apresentar maior gasto de energia elétrica. Já o rendimento foi calculado pela curva característica do motor, usando o índice de carregamento como dado de entrada.

TABELA 4. Características técnicas de bombas e de motores elétricos com e sem acréscimos na potência em função da vazão para pressão de $392 \mathrm{kPa}(40 \mathrm{mca})$. Specifications of pump and electric motors with and without increases in power as a function of the flow for a pressure of $392 \mathrm{kPa}(40 \mathrm{~m})$.

\begin{tabular}{|c|c|c|c|c|c|c|c|c|c|}
\hline 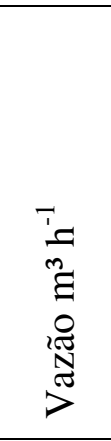 & 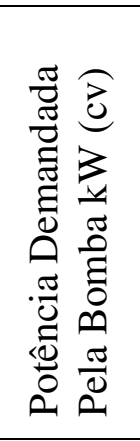 & 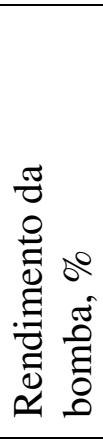 & $\begin{array}{l}\frac{0}{0} \\
0 \\
0 \\
0 \\
0 \\
0 \\
0 \\
\vdots \\
z\end{array}$ & $\begin{array}{l}0 \\
\frac{0}{0} \\
\sum_{i}^{0} \\
\sum\end{array}$ & $\begin{array}{l}\text { Potência } \\
\text { Nominal } \\
\text { do Motor } \\
(\mathrm{kW})\end{array}$ & $\begin{array}{c}\text { Índice } \\
\text { de } \\
\text { Carregamento } \\
(\%)\end{array}$ & $\begin{array}{c}\text { Rendimento } \\
\text { do } \\
\text { Motor } \\
(\%)\end{array}$ & $\begin{array}{c}\text { Potência } \\
\text { Mecânica } \\
\text { Disponível, } \\
\text { Incluindo } \\
\text { FS } \\
(\mathrm{kW})\end{array}$ & $\begin{array}{c}\text { Potência } \\
\text { Elétrica } \\
\text { Útil } \\
\text { Necessária } \\
\text { ao Sistema } \\
\text { na } \\
\text { Condição } \\
\text { Nominal } \\
(\mathrm{kW})\end{array}$ \\
\hline \multirow{2}{*}{3,1} & 0,75 & \multirow{2}{*}{46,1} & \multirow{2}{*}{4} & $1 *$ & 0,75 & 100,0 & 82,7 & 0,85 & 0,89 \\
\hline & $(1,0)$ & & & $2 *$ & 1,1 & 66,7 & 79,1 & 1,27 & 0,93 \\
\hline \multirow{2}{*}{7,2} & 1,5 & \multirow{2}{*}{53,7} & \multirow{2}{*}{4} & 1 & 1,5 & 100,0 & 84,2 & 1,69 & 1,75 \\
\hline & $(2,0)$ & & & 2 & 2,2 & 66,7 & 81,8 & 2,54 & 1,80 \\
\hline \multirow{2}{*}{22,8} & 3,7 & \multirow{2}{*}{67,7} & \multirow{2}{*}{2} & 1 & 3,7 & 100,0 & 87,6 & 4,23 & 4,20 \\
\hline & $(5,0)$ & & & 2 & 5,5 & 66,7 & 87,3 & 6,35 & 4,22 \\
\hline \multirow{2}{*}{51,9} & 7,5 & \multirow{2}{*}{76,9} & \multirow{2}{*}{4} & 1 & 7,5 & 100,0 & 90,0 & 8,46 & 8,18 \\
\hline & $(10,0)$ & & & 2 & 9,2 & 80,0 & 91,6 & 10,58 & 8,03 \\
\hline \multirow{2}{*}{107,9} & 15,0 & \multirow{2}{*}{79,9} & \multirow{2}{*}{2} & 1 & 15,0 & 100,0 & 90,4 & 16,93 & 16,28 \\
\hline & $(20,0)$ & & & 2 & 18,5 & 80,0 & 90,8 & 21,16 & 16,21 \\
\hline \multirow{2}{*}{704,7} & 90,0 & \multirow{2}{*}{83,5} & \multirow{2}{*}{4} & 1 & 90,0 & 100,0 & 94,4 & 105,80 & 97,46 \\
\hline & $(125,0)$ & & & 2 & 110,0 & 83,3 & 94,9 & 126,96 & 96,94 \\
\hline
\end{tabular}

Nota: Tanto o método 1 quanto o método 2 consideraram motores elétricos com fator de serviço conforme ABNT 7094, ou seja, sempre maiores do que 1,00. (*) Método 1 - Dimensionamento de força motriz sem índices ou fatores de segurança que acrescentem a potência demandada pela bomba. (**) Método 2 - Dimensionamento de força motriz com índice ou fator de segurança que acrescentem a potência demandada pela bomba (BERNARDO et al., 2005).

a) A escolha das vazões foi feita de modo que, para as pressões (alturas manométricas) estudadas, os índices de carregamento dos motores fossem iguais a $100 \%$ pelo método de dimensionamento da força motriz, sem índice ou fatores de segurança que acrescentem a potência demandada pela bomba (método 1). Essa escolha foi feita porque os pontos críticos para avaliação da influência da adoção de fatores de segurança e/ou de acréscimo ocorrem quando os índices de carregamento são máximos. De outra forma, os motores relacionados (com índices de carregamento menores do que 100\%) já teriam (mesmo sem fatores de segurança e/ou consideração de fatores de serviço) alguma potência disponível.

b) As potências demandadas pelas bombas (potências mecânicas no eixo) foram aquelas entre as potências comercialmente disponíveis de motores elétricos.

c) $\mathrm{O}$ rendimento da bomba foi obtido pelo uso do programa computacional de seleção de bombas centrífugas AGRIBOMBAS (2000). Observou-se que o rendimento das bombas teve tendência de aumento à medida que se elevou a vazão de projeto. Os rendimentos das bombas variaram de $46,1 \%$ a $83,5 \%$. As características da bomba para cada valor de altura manométrica e vazão foram as mesmas do dimensionamento de motores sem e com o uso de fatores de segurança ou acréscimo na potência demandada (método 1 e método 2 , respectivamente). 
d) Considerou-se o mesmo número de polos dos motores nos dois métodos de dimensionamento.

e) A comparação entre as potências nominais dos motores pelos métodos 1 e 2 indicou, claramente, que o método 2 exige motores de potências superiores àquelas exigidas pelo método 1 . Isso implica necessidade de investimento maior quando se utiliza o método 2. Esse investimento maior foi proporcional, também, ao aumento dos custos de comando, proteção, sinalização e condutores para instalação de motores de maior potência. $83,3 \%$.

f) Observou-se que o índice de carregamento dos motores pelo método 2 variou de $66,7 \%$ a

g) Quanto à comparação dos rendimentos dos motores pelo método 1 com o método 2, não se observou tendência alguma. Na comparação dos 23 casos estudados, em nove das vezes, o método 1 forneceu rendimentos superiores; em 13 das vezes, proporcionou rendimentos inferiores àqueles fornecidos pelo método 2 e, em uma vez, não houve diferença.

h) Finalmente, as quatro últimas colunas das Tabelas 3 e 4 apresentam as potências mecânicas disponíveis, usando o fator de serviço (FS) e a potência elétrica útil necessária ao sistema. O conhecimento da potência elétrica útil demandada foi importante para o cálculo da demanda de potência utilizada em tarifas de energia elétrica, como o sistema horo-sazonal, tarifa azul, ou tarifas para irrigação noturna.

\section{Análise econômica}

A análise econômica considerou os preços dos motores, o consumo e a demanda de energia elétrica. Os preços dos motores foram obtidos pela diferença entre os motores a serem usados nas mesmas situações de projeto.

TABELA 5. Comparação dos gastos com energia elétrica nos dois métodos propostos para a pressão de $392 \mathrm{kPa}$ (40 mca) e 4.000 horas de funcionamento anual. Comparison of energy expenses in the two proposed methods for the pressure of $392 \mathrm{kPa}(40 \mathrm{~m})$ and 4,000 hours of annual operation.

\begin{tabular}{cccccc}
\hline \multirow{2}{*}{ Potência Nominal dos Motores } & \multicolumn{2}{c}{$\begin{array}{c}\text { Energia } \\
\mathrm{kWh} \mathrm{ano}^{-1}\end{array}$} & \multicolumn{2}{c}{$\begin{array}{c}\text { Gasto com Energia Elétrica } \\
\mathrm{R} \$ \text { ano }^{-1}\end{array}$} \\
\hline Método 1 & Método 2 & Método 1 & Método 2 & Método 1 & Método 2 \\
0,75 & 1,10 & 3.558 & 3.722 & 193,37 & 202,28 \\
1,10 & 1,50 & 5.318 & 5.270 & 289,03 & 286,41 \\
1,50 & 2,20 & 6.976 & 7.198 & 379,15 & 391,21 \\
2,20 & 3,00 & 10.182 & 10.270 & 553,37 & 558,16 \\
3,70 & 5,50 & 16.797 & 16.861 & 912,93 & 916,42 \\
5,50 & 7,50 & 24.658 & 24.479 & $1.340,17$ & $1.330,43$ \\
7,50 & 9,20 & 32.725 & 32.140 & $1.778,62$ & $1.746,79$ \\
11,00 & 15,00 & 48.910 & 49.176 & $2.658,25$ & $2.672,71$ \\
15,00 & 18,50 & 65.130 & 64.846 & $3.539,80$ & $3.524,37$ \\
37,00 & 45,00 & 157.256 & 157.602 & $8.546,87$ & $8.565,65$ \\
90,00 & 110,00 & 389.907 & 387.777 & $21.191,46$ & $21.075,66$ \\
\hline
\end{tabular}

Nota: Os custos de energia elétrica foram obtidos pela energia e demanda com os valores de $\mathrm{R} \$ 0,026$ e $\mathrm{R} \$ 9,45$, respectivamente.

Na Tabela 5, mostram-se, para os dois métodos, o consumo e a demanda de energia com os gastos totais com energia elétrica para a pressão de $392 \mathrm{kPa}(40$ mca) e 4.000 horas de funcionamento anual. $\mathrm{O}$ cálculo de demanda e de consumo de energia foi feito considerando-se o índice de carregamento, a potência nominal do motor e o rendimento do mesmo nessa situação. Para isto, as curvas características dos motores fornecidas pelos fabricantes foram consultadas. $\mathrm{Na}$ 
Tabela 5, pode-se observar que nem sempre o aumento na potência do motor, entre os dois métodos, faz diferença no custo final da energia elétrica. Nessa simulação, o aumento de potência entre os motores apresentados para uma mesma função, de $22 \%$ a $46 \%$, não correspondeu às expectativas com a mesma percentagem no gasto total com energia elétrica. Isso pode ser explicado, primeiro, pela escolha dos motores para trabalharem no limite de suas capacidades e, segundo, pelas variáveis correspondentemente inversas, em que um motor de maior potência transmite uma proporcionalidade no índice de carregamento menor no mesmo motor, e a comparação com outro motor corresponde ao contrário, no cálculo final do gasto de energia elétrica.

TABELA 6. Variação dos preços entre motores de 2 e de 4 polos. Price variation among engines of 2 and 4 poles.

\begin{tabular}{ccccc}
\hline \multirow{2}{*}{\begin{tabular}{c} 
Potência \\
Nominal do Motor \\
\cline { 2 - 5 }$(\mathrm{cv})$
\end{tabular}} & $\begin{array}{c}\text { Preço do Motor } \\
(\mathrm{R} \$)\end{array}$ & $\begin{array}{c}\text { Variação } \\
(\%)\end{array}$ & $\begin{array}{c}\text { Preço do Motor } \\
(\mathrm{R} \$)\end{array}$ & $\begin{array}{c}\text { Variação } \\
(\%)\end{array}$ \\
\hline 1,0 & 183,36 & 13,0 & 197,85 & 13,2 \\
1,5 & 207,15 & 11,4 & 223,87 & 22,3 \\
2,0 & 230,84 & 18,8 & 273,75 & 21,4 \\
3,0 & 274,34 & 34,7 & 332,35 & 30,4 \\
4,0 & 369,44 & 17,3 & 433,44 & 7,2 \\
5,0 & 433,24 & 20,2 & 464,78 & 19,5 \\
6,0 & 520,55 & 12,2 & 555,32 & 8,4 \\
7,5 & 584,20 & 23,4 & 601,92 & 26,4 \\
10,0 & 720,64 & 20,7 & 760,81 & 18,2 \\
12,5 & 869,75 & 9,5 & 899,38 & 12,4 \\
15,0 & 952,22 & 18,8 & $1.010,99$ & 19,4 \\
20,0 & $1.131,57$ & 40,2 & $1.206,98$ & 34,8 \\
25,0 & $1.586,35$ & 6,7 & $1.627,49$ & 25,8 \\
30,0 & $1.692,81$ & 50,4 & $2.047,60$ & 27,4 \\
40,0 & $2.546,66$ & 8,2 & $2.607,64$ & 10,0 \\
50,0 & $2.754,26$ & $* 72,9$ & $2.867,93$ & 62,7 \\
60,0 & $4.762,56$ & 7,9 & $4.665,24$ & 13,7 \\
75,0 & $5.136,80$ & 31,0 & $5.304,51$ & 18,5 \\
100,0 & $6.728,35$ & 41,4 & $6.284,28$ & 42,7 \\
125,0 & $9.514,82$ & 13,1 & $8.968,73$ & 18,5 \\
150,0 & $10.761,69$ & 24,6 & $10.627,42$ & 21,6 \\
175,0 & $13.407,21$ & 2,1 & $12.920,23$ & $* 1,1$ \\
200,0 & $13.692,90$ & 53,4 & $12.784,25$ & 25,6 \\
250,0 & $21.005,02$ & - & $16.057,62$ & - \\
\hline
\end{tabular}

Fonte: WEG (2000).

Analisando a Tabela 6, verifica-se que a diferença nos preços dos motores, para o primeiro ascendente, apresenta ganho entre $1,1 \%$ e $72,9 \%$, que pode representar uma decisão na escolha do tipo e da potência do motor na hora da compra.

Na Tabela 6, mostra-se o preço dos motores de 2 e de 4 polos, bem como a variação percentual de preços entre modelos de potências disponíveis comercialmente. Por exemplo, para motores de 2 polos, o modelo de $1,5 \mathrm{cv}$ é $13 \%$ mais caro do que o de $1,0 \mathrm{cv}$.

\section{CONCLUSÕES}

A obrigatoriedade de os motores elétricos terem fatores de serviço de, no mínimo, 1,15 (Tabela 1) poderá atender, na maioria das vezes, às condições de trabalho não previstas (curvas características de bombas, condições que não correspondam às especificações, oscilação excessiva e 
ao desequilíbrio dos sinais de tensão), que requerem fatores de segurança ou de acréscimo na potência demandada.

A utilização acumulada de fatores de segurança e de acréscimo de potência no dimensionamento de motores, para acionamento de bombas hidráulicas, implicou, em um número significativo de vezes, rendimento inferior aos dos mesmos sistemas quando calculados sem esses fatores.

É necessário que não somente motores elétricos, mas também bombas hidráulicas tenham certificado de suas curvas características emitido por agente credenciado pelo órgão competente e que se trabalhe com o objetivo de assegurar sinais elétricos de melhor qualidade, incluindo nível e oscilação de tensão e de frequência.

Quanto mais se consegue descrever o equipamento, seja o motor, a bomba, seja o sinal elétrico, menor a necessidade de superdimensionamento. Os motores estudados apresentaram excesso na potência disponível entre $15 \%$ a $88 \%$ da potência demandada do conjunto, significando motor com folga excessiva.

Este trabalho foi desenvolvido para verificar e adequar equações no dimensionamento de motores para bombeamento de água para irrigação que visam à otimização do consumo de energia elétrica.

\section{AGRADECIMENTOS}

Os autores são gratos pelo apoio financeiro da CAPES, Coordenadoria de Apoio ao Pessoal de Ensino Superior, e ao CNPq, Conselho Nacional de Desenvolvimento Científico e Tecnológico.

\section{REFERÊNCIAS}

ABNT. ASSOCIAÇÃO BRASILEIRA DE NORMAS TÉCNICAS. NBR 7094: máquinas elétricas, motores de indução, especificação. Projeto original NBR 7094, 1994. Rio de Janeiro, 1996.

AGRIBOMBAS. Programa para seleção de bombas agrícolas, versão 1.0: uso racional de energia na agricultura. Belo Horizonte: CEMIG, 2000. Disponível em: <http://www.cemig.com.br $>$. Acesso em: 11 jul. 2008.

BERNARDO, S.; SOARES, A.A.; MANTOVANI, E.C. Manual de irrigação. 7.ed. Viçosa: Imprensa Universitária, 2005. 611 p.

ELETROBRÁS. Guia operacional de motores elétricos. Rio de Janeiro: PROCEL, 1998. v.1, $161 \mathrm{p}$.

SANTOS, W.L. Desenvolvimento de uma metodologia para representação analítica de curvas características de bombas hidráulicas visando à sua seleção, ao seu dimensionamento e à simulação de sua operação. 2001. 698 f. Tese (Doutorado em Engenharia Agrícola) - Departamento de Engenharia Agrícola, Universidade Federal de Viçosa, Viçosa - MG, 2001.

WEG. Catálogo eletrônico 2000. Jaraguá do Sul. Disponível em: <http://www.weg.com.br>. Acesso em: 19 jul. 2008. 